\title{
Decreased Business Uncertainty by Using Bayesian Networks for the Paradigm Shift in Business Simulator
}

\author{
Alberto Ochoa ${ }^{1}$, Miguel Ruiz-Jaimes ${ }^{2}$, Sandra Leon ${ }^{2}$, \\ Yadira Toledo², Iván Ramírez ${ }^{2}$ \\ ${ }^{1}$ Juarez City University, Ciudad Juárez, \\ Mexico \\ ${ }^{2}$ Universidad Politécnica del Estado de Morelos, Morelos, \\ Mexico
}

\begin{abstract}
Most small businesses related with Textile Industry or clothes in Latin America fail in his first two years this due to lack of follow-appropriate decisions to bring out various problems, which is why the business simulators help lessen the burden of analyzing the right decision-making in future scenarios and have a high complexity due to exogenous events. To implement a business Simulator for SMEs and in particular in the marketing segment, it is necessary to know the main variables that affect the decisions that should be taken as necessities in any business, and especially when it's an SME that is about to begin its operations. Strategies as the use of Bayesian networks are associated with the behaviors are linked with the variables and scenarios that can be a presenter during the life of the business.
\end{abstract}

Keywords: Business simulator, Exogenous variables, Marketing to detail, Decision based on strategies.

\section{Introduction}

On the model of the marketing, variables shall be taken into consideration more important in making decisions, and which are also linked to the different modules that must have the Simulator business for the University's incubator. Business Simulator encompasses each of the disciplines of management, however, the investigation of the document in specific fits in the field of marketing, which is the focus of the module to develop. About the module of marketing should be considered and specified that it is of utmost importance since the marketing consists of a set of principles and practices that are carried out with the aim of increasing trade, and especially the demand. The concept also makes reference to the study of the procedures and resources that pursue the same aim. The marketing involves the analysis of commercial enterprises management. Its intention is to withhold and retain customers' actuals having an organization, while trying to add new buyers. 


\section{Methodology Used: Bayesian Networks}

Bayesian networks may be constructed by applying a learning algorithm to the data set that allows learning a structure. The greatest potential for them resides in the ability to combine expert knowledge with empirical data.

Other benefits of these networks include:

- Ability to reflect causal relationships,

- Running the model with incomplete data and assumptions,

- Forward and backward reasoning,

- Modelling uncertainty,

- Combination of quantitative and qualitative data.

We could define a Bayesian network as a set of variables coded probabilistically, a graphical structure that connects these variables in terms of relationships of conditional independence and a set of distributions susceptible of conditional probability that can be modified based on evidence by means of Theorem of Bayes' theorem. One of the most important advantages of Bayesian networks is that they can represent both the quantitative aspect of a problem as its qualitative aspect. The theoretical support of the qualitative dimension in Bayesian networks is provided by the graph theory. Graph theory tries to create graphical models (graphs) that represent the elements of the problem in a holistic sense and was introduced by Euler to solve the Seven Bridges of Königsberg.

\section{Advantages of Bayesian Networks}

The most important advantage offered by Bayesian networks over other methods of multivariate analysis that can represent unison qualitative and quantitative dimension of a problem in an intelligible graphical environment. Another important advantage of Bayesian networks is that they can work with lost data efficiently, which in practice is desirable (e.g. highlighted by [2.3.4]). However, a disadvantage of missing cases is that if a state of the variable appears the probability for this is zero. Also, reduce the envelope setting data [6] and as Bayesian statistical technique, combining prior knowledge about the problem that we study with experimental data [11]. Bayesian networks also allow you to discover the underlying causal structure in a dataset (as is analyzed in: $[4,7,9,11,16])$. This means we can build a probabilistic graphical model from a database containing a set of observations on a set of variables. Bayesian networks have advantages over classical rule-based expert systems are to be used when, for example, for decision-making. First, Bayesian networks represent all information in a single format (probabilistic and graphic) making simple interpretations, allow recant conclusions obtained previously and are no longer reasonable in light of new evidence, they give us a general problem vision, generate a set of ordered alternatives and facilitates the explanation of findings [8]. On the other hand, when we build a Bayesian from expert knowledge to use in counseling in a decision-making network, assigning probabilities (or generation) is simpler [7, 12]. In addition, Bayesian networks allow working with concepts of decision theory as value or expected value against decision 
problems [7, 8, 13]. In the context of inference, Bayesian networks allow bidirectional inferences; that is, from effects to causes and from causes to effects. And what is more interesting, they allow performing abductive inferences; that is, find the best explanation for a set of data $[9,11]$. However, the most important advantage of a Bayesian network in the field of statistical inference is derived from its ability to perform local computations $[14,19]$. This property allows updating probabilities that are carried out efficiently without having to calculate all possible combinations between all levels of the variables (which would mean an exponential increase in the calculations as the number of variables in the model or number of states by variable) when incorporated determined knowledge to the graphic structure. Thanks to this property derived from the principles of dependence and conditional independence, Bayesian networks are tools particularly suited for tasks that require rapid and continuous updating control processes, e.g. industrial. Another important advantage of Bayesian networks is that they can work with lost data efficiently, which in practice is desirable (e.g. highlighted by [9, 11, 15, 17]). However, a disadvantage of missing cases is that if a state of the variable appears the probability for this is zero. Also, reduce the envelope setting data [9] and as Bayesian statistical technique, combine the foreknowledge that we respect to the problem of study with experimental data [16]. In [18], Bayesian networks are statistical oriented tools for causal modeling. A Bayesian network is defined by two main components: a qualitative based on graph theory and other quantitative based on probability theory. These tools have been used for prediction, classification and diagnosis through the use of Bayes' theorem as a heuristic updater belief propagation algorithms and probability. However, although the survey methodology has been used to illustrate the automatic construction of Bayesian networks, there are not many jobs of this kind. We show the process of building Bayesian networks from data from a survey of entrepreneurial attitudes in a sample of university students. Bayesian networks (also known as probabilistic causal networks, Bayesian expert systems, probabilistic expert systems, causal networks, belief networks and influence diagrams) are statistical tools oriented modeling, qualitative dimension of a Bayesian network is based on the theory graphs. Although the definition of graph may vary according to the authors (e.g. $[13,15,16,17$, 20]), a graph can be defined as a set of nodes (variables or vertices) and a set of edges (or links). However, a Bayesian network is a special type of graph, or what is known as a directed acyclic graph (or DAG). In a DAG edges or bonds linking the variables they are oriented and are plotted as arrows. The directed links indicate a Bayesian network causal influence or dependence between variables. Thus, the graph $\mathrm{A} \rightarrow \mathrm{B}$ indicates direct influence on B; or what is the same, that B depends on A. In causal terms, we would say that "A causes B" or "B is the effect of A". In a Bayesian network encodes the graphical relationships of dependence and conditional independence between variables that are used to expedite the update probability and statistical inference. In the quantitative dimension, a Bayesian network consists of three major elements: the concept of probability as a degree of subjective belief on the occurrence of an event, Bayes' theorem as a heuristic updater beliefs and a set of conditional probability functions. The probability can be understood, at least four ways: classically, as relative frequency when trials tend to infinity, axiomatically or subjectively. The first three are compatible and have been encompassed under the name 
of classical perspective of probability or frequentist and often counter the fourth has been called Bayes (i.e. [2, 7, 9, 11, 13, 18]). Bayesian networks use Bayes' theorem to update the model of probabilities. For example, suppose we have a model that represents several variables and each has an associated probability of occurrence in each of their states. If we know the state of one Bayes theorem it recalculates all probabilities the model to be consistent with this evidence and spreads throughout the network via the links between nodes. Finally, for the Bayes theorem to operate must exist, one for each variable, a set of probability functions encoding relations' probabilistic dependence of each variable and all the variables on which it depends. In particular, there must be a table of conditional probability (TCP) representing the probability of each state variable depending on the variables on which it depends. Computer programs that work with Bayesian networks are designed so they can learn the graphical structure and the implicit stochastic structure in a dataset. That is, build a (qualitative and quantitative) Bayesian network from a database. There are different procedures aimed at achieving this objective (see, for example, $[3,8$, 10]), and although many have used survey data to illustrate the automatic construction of Bayesian networks not many networks built from surveys. Finally, indicate that Bayesian networks are a type of statistical tool rarely used in survey research and we believe that this methodology could benefit from its use, either through automatic creation procedures, or through expert judgment [4].

\section{Construction of Bayesian network from data}

There are several techniques for building Bayesian networks from a database. In this research, we have used the algorithm K2 [3] and stochastic variable neighborhood search ([16]). We passed briefly describe these algorithms. See Figure 1.

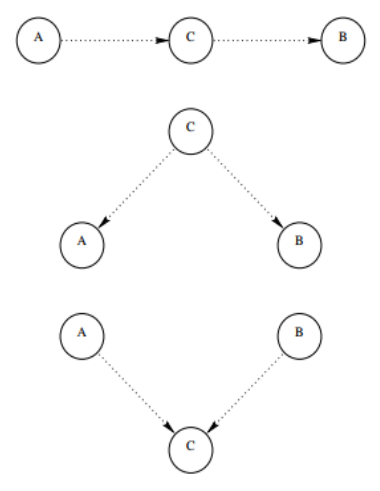

Fig. 1. Graphic characterization of the concept of d-separation

\section{Applications of Bayesian networks to software engineering}

Bayesian networks are becoming increasingly popular in engineering, artificial intelligence and statistical time. They have been successfully applied to domains such as medicine, risk 
assessment, vision, diagnostics systems and networks, fraud detection, spam, etc. [11]. In software engineering Bayesian networks, have been used in different areas such as:

Estimation of effort and quality. In [4] is provide a critical review of the methods available in the literature for predicting defects, arguing that models based on size and complexity cannot predict efficiently. The authors state that the incorporation of Bayesian networks taking into account such factors as the ability of programmers / analysts, complexity of design and the methods and procedures used generates more accurate predictions. They have shown that is possible to introduce process attributes and product attributes in a Bayesian network. [18]) compared the predictions of Naïve Bayes classifier to estimate the effort of software projects with other techniques of data mining and regression trees and neural networks, showing that such a simple technique as naïve Bayes has the potential to it is used as a possible technique viable estimate

- Testing software. Author of [20] discuss how Bayesian networks can be used as a testing tool, with questions like "what if ..." that helps managers and staff responsible for testing in decision-making.

- Reliability. Fenton et al. have carried out several projects, such as DATUM (Dependability Assessment of safety critical systems through the Unification of Measurable) [9], SERENE (Safety and Risk Evaluation using Bayesian Networks) in which networks have been developed to estimate the reliability and security of systems in various fields [7].

- Graphics and user interaction interfaces. As an example, the Lumiere project [12] of Microsoft investigated ways to improve the interaction between users and software using Bayesian networks. He served as the basis for Microsoft Office wizard when the user used the aid, since the inference engine considers the Bayesian network user actions, application events and user profiles. They have also been used as diagnostic aides for detecting printing problems, etc.

\section{Properties and limitations of Bayesian networks}

Bayesian networks have a number of features that make them suitable for software engineering:

- Graphic representation. By their nature, Bayesian networks provide a graphical representation of the explicit dependency relationships domain. Generally, the variables in software engineering such as effort or cost are influenced by many factors. Bayesian networks allow us to model complex systems allowing us to understand the causal relationships graph displaying them through.

- Qualitative and quantitative modeling. Bayesian networks are formed by a qualitative component, the graph, and a quantitative part, the probability tables, which allow using objective criteria (for example, using completed projects) and subjective (e.g. using beliefs domain experts.

- Bidirectional Inference. Bayesian inference networks can do both ways, i.e., the input variables can be used to predict the output variables and vice versa. Setting the output variables with desired values, it is possible to predict which values of the input variables 
allow such output. For example, using inference forward, one can predict the final number of defects based on variables such as project size, complexity, design effort, effort in testing, etc. On the contrary, we could fix a number of defects and effort needed to predict which satisfies said output.

- Sensitivity analysis. Given a set of evidence, Bayesian networks allow easily calculate the sensitivity of certain variables, simply by modifying the evidence.

- Uncertainty. Bayesian networks can model degrees of certainty rather than exact values. Therefore, allow modeling uncertainty effectively and explicitly way, so they can make good predictions with incomplete information. In fact, [11] estimates claim that software engineering is a probabilistic assessment of a future event, and is therefore the reason that project managers do not obtain good results.

- Values of confidence. The output of a Bayesian network is a probability distribution instead of unique values. This type of information can be used to measure the confidence we can place in the output of the Bayesian network, which is essential if the model will be used in decision-making. For example, in variable low, medium, and high states, the Bayesian network estimates the probability of each of the states [16].

\section{Applications}

\begin{tabular}{|l|l|}
\hline Utilization & Research Field \\
\hline Bayesian networks are important for psychology in two ways: & Psychology \\
economically and scientifically. Economically Bayesian networks & \\
could generate a market of "elicitation of probabilities" oriented \\
development of expert systems where psychology could play a \\
key role. The task for psychology in this regard would guide the \\
experts on how to make appropriate judgments likely trying to \\
overcome the biases that people usually incurred when \\
performing such tasks. \\
\hline $\begin{array}{l}\text { A particularly case: Prostate cancer is the most common } \\
\text { malignancy among men over 50 years of age and the second }\end{array}$ & Medicine \\
leading cause of cancer death (lung cancer is first). The \\
probability of recovery depends on the stage of the cancer \\
(whether it is located exactly in the prostate or has spread to other \\
parts of the body) and the patient's health in general. Therefore, it \\
is important to diagnose at an early stage. However, symptoms of \\
prostate cancer are very similar to those of benign prostatic \\
hyperplasia (BPH) or other prostate problems, so it is easy to \\
confuse. Therefore, it is useful to have a tool that helps the doctor, \\
especially general practitioners, to make a differential diagnosis \\
of possible diseases based on their probabilities. \\
The use of tools to make the design of a Bayesian network is \\
paramount, is why referring to the above introduction, we will talk \\
about some of them.
\end{tabular}




\section{Problem Statement}

In an economic system is the growth engine of the company, in different sizes, ratings, and economic activity in the preceding paragraphs is justified because in this case study interested SMEs. However, they present some difficulties according to $[11,20]$ :

- Lack of organization and management.

- Insufficient technology for new challenges.

- Staff turnover due to lack of selection processes.

- Disability credit for lack of collateral.

- Problems capital.

- Business model and inefficient operation.

- Limited to financing at reasonable rates of interest Access.

- Do not reinvest their profits for improving machinery and equipment.

The problem is multifactorial and it depends on is staying within its respective industry, for example, specialized studies of the National Commission for the Protection and Defense of Financial Services Users (CONDUSEF) indicate that only half of existing SMEs in the country accounted survive for eighteen months [20], in this regard the difficulties financial reasons are skewed because 80 percent of SMEs who die for lack of financial management [11].

The effect disappears an SME, it is certainly a failure for the entrepreneur, but the effect is greater for the family that depends on this and goes without a source of income. The National Employment Service in its statistics records mention that for an unemployed person to find work again, time is of one to six months. Of course without considering that this should be permanent and well paid, the latter under the present conditions of our economy can be a Utopia.

According to [13], there are many factors that put their stability and survival at risk, with the absence of a strategic business plan that primarily impacts the growth and permanence of an SME. Therefore, some of the mistakes that commonly make are:

- Lack of institutionalization of their organizational values

- Debts and obligations assumed by shareholders in a personal capacity

- Inadequate segregation of duties

- Technological Backwardness

- Lack of reinvestment of profits in working capital

- Lack of trained personnel

- Lousy service to its customers

- And, therefore, problems with the tax authorities

However, we cannot generalize, because at the time no doubt the particular reality of each company is different and depending on their economic activity to play, so it is of 
importance to make a diagnosis to identify the factors of business competition according to the industry you choose. One factor resulting in lack of success of a business is the case of entrepreneurs who start in reverse, i.e., they put the business based on what they know or like to do, without considering whether that product or service will cover a need market, it sometimes ends up winning the dealer, who identified the need to market, rather than the producer [11].

Also, the lack of business, i.e., many start their business because they have no other, and that prevents them gauge the integrity and scope of its business. Adapting to changes in the environment is another key difficulty of SMEs does not have a vision or reading systematic risk. Among the factors leading to delay a determination, especially in SMEs, it emphasizes paying little attention to the behavior of markets and the environment [18]. The ability of the owners to adapt to changes in their environment does not depend on the size of the company, strategically it should go out to his vision and mission to become more competitive and stay in their economic activity.

And according to [8], SMEs can also have certain advantages over others:

- Personalized service: As a small company, worries and occupies more customer satisfaction, usually a worker treats all customer purchase process.

- Improved access to market information: For the size and proximity of your target audience, get first-hand information about political and economic developments that could affect it so you can improve your processes.

- Flexibility tastes and preferences: You can easily adapt to the requirements of their customers or simply patronizing your customers.

- Commitment of employees: Having a single organization, it is easy for workers to contribute new ideas for production or management that facilitate the operation of the company and that these ideas are applied causing workers feel motivated. Unlike large companies is difficult a change in structure.

- Better handling of complaints: As personalized attention, you can try a worker to resolve the claim or complaint from a customer, ensuring the satisfaction of this.

- Improved ability to correct errors: Because of the size and structure of these businesses, it is easier to correct errors they have.

- Faster and timely decisions: For its simple and little hierarchical structure, the ability to make decisions is more optimal and timely.

- Allow the creation of value and in the future better decision-making.

It is the result of a profound and serene reflection by the investigator after reviewing the relevant literature (theoretical and empirical background) detail and internalized the main concepts and theoretical propositions that allow you to formulate clearly and domain the problem to be solved with the investigation. The growth of the national economy as through System Cyclical indicators in the last 35 years and considering the respective federal governments has the following behavior: 


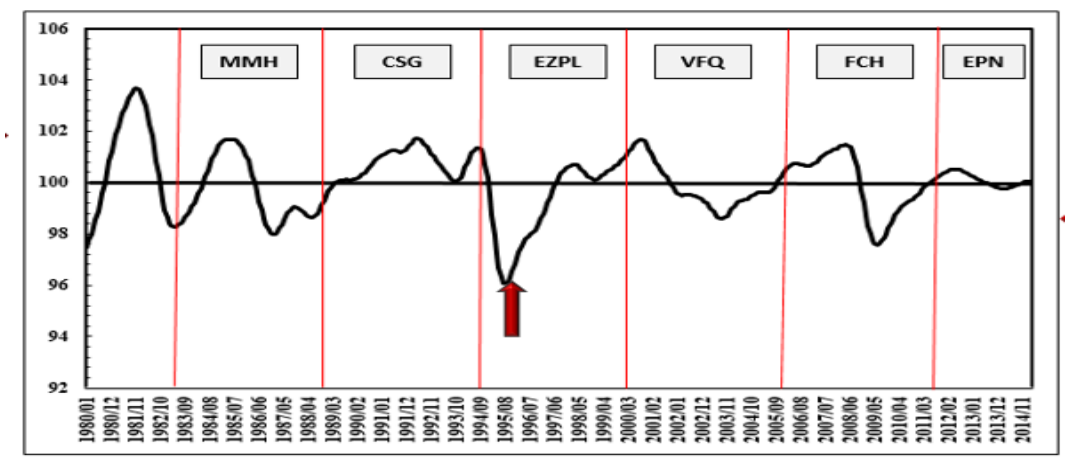

Fig. 2. The growth cycle in Mexico. Cyclical system Indicators: 1980 to 2015. Source: Taken from: The cycle 1980.1-2015.1 national economic growth in the context of the economic policy of neoliberal [11].

Note for Fig. 2:

MMH.- Miguel de la Madrid Hurtado,

CSG.- Carlos Salinas de Gortari,

EZPL.- Ernesto Zedillo Ponce de León,

VFQ.- Vicente Fox Quesada,

FCH.- Felipe Calderón Hinojosa,

Enrique Peña Nieto EPN.

\section{Models of the interfaces}

In the Simulator's business, as first instance will be specifically in the module of marketing the General information of the company as the name, the commercial rotation, many employees and above all the initial investment, this with the purpose of having a control according to the strategies and options that are available as a tool.

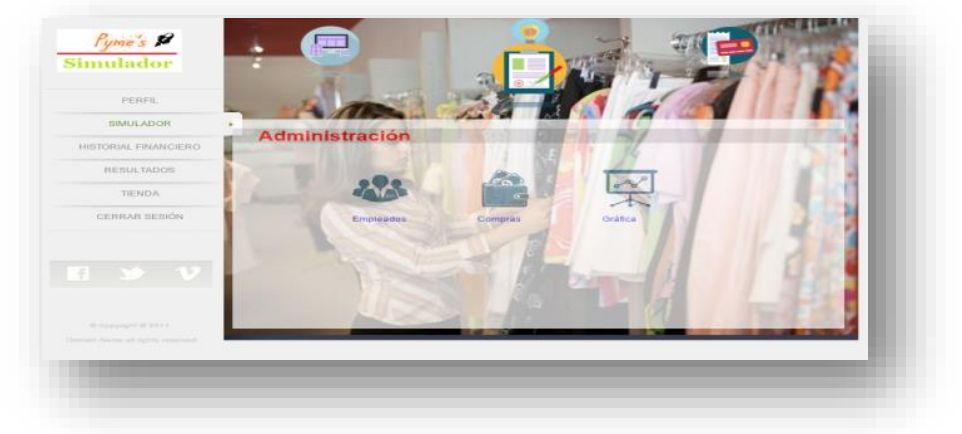

Fig. 3. Main screen simulator contains 4 modules that mimic the behavior of the company, which in turn contains sub-modules where the player will take the decisions of the company 


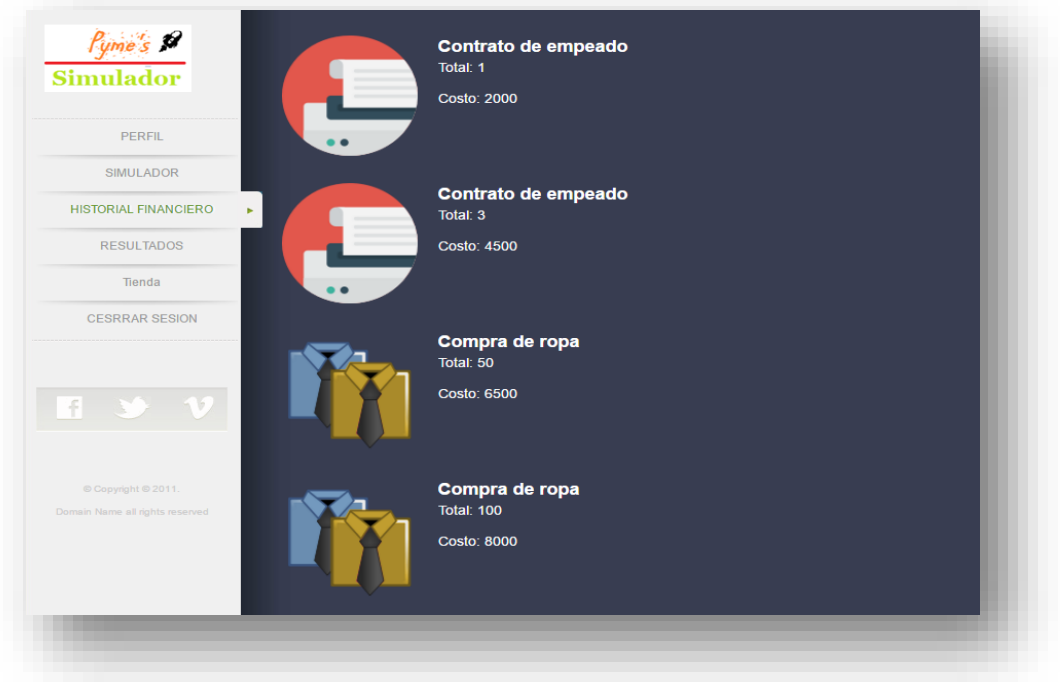

Fig. 4. History of all movements, purchases, contracts, and monitoring the management of the company

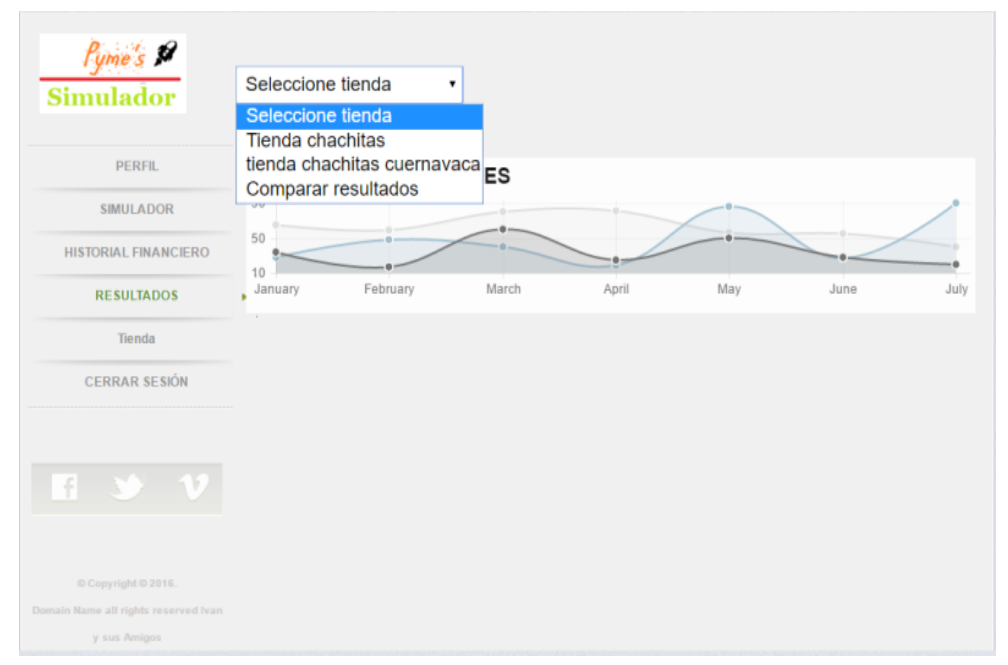

Fig. 5. May compare the results of different companies, either individually analyze the result of decisions by a graph.

The simulators are mostly computer programs that are using a programming language. The algorithm of the program design are models that are starting to define a number of 
relevant internal, external variables and the possible interactions between these, which should allow to simulate the operation [3].

Therefore, it is important to have well-defined strategies, so that there are no variables that are not used in the design of the program. Module marketing that is aimed at promoting and advertising the business serve as a simulator guide before starting operations and that this allowed mistakes, while they still cost.

\section{Conclusions and Future Research}

Module marketing that is aimed at promoting and advertising the business serve as a simulator guide before starting operations and that this allowed mistakes, while they still cost. A business simulator is a learning tool that is intended to play an economic, financial or business system. This is given a virtually, allowing you to participate in the decisionmaking process and in the process of management of a company. Business Simulator gives guideline to learn without any risk, but is empirical, acquired knowledge of what is and is not for. In [1] is considered that a business simulation is a creation of a business for the purpose of experiment and learn real environment through new experiences, where the participant assumes a role and must make decisions that impact on the environment, the situation and the results of the company as is analyzed in [12]. Therefore, comes to the conclusion that is indispensable in the field of the incubator of the diverse government systems, where in addition to a theorist of the SME study, in a simulator that lasts approximately 8 weeks' equivalent to 4 years (real time), since they are in practice knowledge and aware of the consequences of decisions taken without any risk.

The advantages of simulators are:

- You can learn the result of decisions taken in a prior period. This leads to the competitive advantage of information before acting.

- Allows you to know the effect of implemented marketing strategies.

- Allows you to measure and evaluate the results achieved in the performance together with each of the modules.

- Develops the ability to make decisions, carry out strategies and correct unforeseen.

- Allows you to apply and develop knowledge in the field.

- Facilitates the panorama of the scenarios that may occur in the company.

Precisely in the marketing module you can interact with the strategies of low-cost, since a SME to initiate operations must have a backup of at least 4 months of salaries, expenses, etc. While it is unveiled, and attract customers, however, not so should be put to one side the advertising and promotion. To the study analyzed un [10] state that: The marketing concept is based on the definition proposed by the United States Marketing Association (American Marketing Association), which expresses that it is the process of planning and executing the conception, price, promotion and distribution of ideas, goods and services to create exchanges that satisfy individual and organizational objectives. The key is the 
concept of Exchange, in which someone renounces something to receive anything you need or want, which should happen as follows:

a) Have at least two parties involved.

(b) Each party must have something of value to the other.

(c) Each party must be able to communicate with each other and provide goods or services to such party.

(d) Each party must be free to accept or reject the offers of the other.

(e) Each party must believe that it is appropriate or desirable to deal with the other, as is shown in [13].

The job of marketing is essential today and is useful in business simulators.

\section{References}

1. Blanque, S., Odriozola, J.: Simuladores: Herramienta de apoyo para el aprendizaje del marketing. Facultad de Ciencias Económicas, Universidad Nacional del Nordeste, Buenos Aires, Argentina, pp. 2-8 (2010)

2. Cowell, R. G.: Parameter learning from incomplete data for Bayesian networks. In: AISTATS (1999)

3. Edwards, W.: Hailfinder. Tools for and experiences with Bayesian normative modelling. American Psychologist, 53, 416-428 (1998)

4. Edwards, W., Fasolo, B.: Decision technology. Annual Review of Psychology, 52, 581-606 (2001)

5. González, E., Cernuzzi, L.: Apoyando el aprendizaje de habilidades empresariales mediante la utilización de un simulador. En: J. Sánchez (Ed.), Nuevas Ideas en Informática Educativa, Volumen V, pp. 8-19, 23-35, Santiago de Chile (2009)

6. Harary, F.: Graph Theory. Addison-Wesley, Reading, MA (1969)

7. Heckerman, D.: A Bayesian approach to learning causal networks. In: Proceedings of the Eleventh conference on Uncertainty in artificial intelligence, pp. 285-295, Morgan Kaufmann Publishers Inc. (1995)

8. Howard, J., Sheth, J.: Prespectives in Consumer Behavior. Grenvich, Scott Foresman (1969)

9. Huete, J. F.: Sistemas expertos probabilísticos: modelos gráficos (1998)

10.Jansen, R., Yu, H., Greenbaum, D., Kluger, Y., Krogan, N. J., Chung, S., ... Gerstein, M.: A Bayesian networks approach for predicting protein-protein interactions from genomic data. science, 302(5644), 449-453 (2003)

11.Lamb, C., Hair, J., McDaniel, C.: Principles of marketing. $2^{\mathrm{a}}$ ed., Publishing Co. USA: SouthWestern (1994)

12.Maciá, M. A., Barbero, M. I., Pérez-Llantada, M. C., Vila, E.: Psicología y teoría de la decisión: aplicaciones. Madrid: Universidad Nacional de Educación a Distancia (1990)

13.Mussons, J., Comajuncosa, J.: Nuevas técnicas de formación e investigación empresarial mediante el desarrollo de un simulador de negocios complejo en entorno real. Universidad Politécnica de Cataluña, Campus Nord/Manresa, Barcelona, Cataluña, España (2005)

14.Nadkarni, S., Shenoy, P. P.: A causal mapping approach to constructing Bayesian networks. Decision support systems, 38(2), 259-281 (2004)

15.Neapolitan, E. R., Morris, S.: Probabilistic modelling with bayesian networks. The SAGE handbook of quantitative methodology for the social sciences, 371-390 (2004) 
Decreased Business Uncertainty by Using Bayesian Networks for the Paradigm Shift in Business ...

16.Palomo, M.: Los procesos de gestión y la problemática de las Pymes. Ingenierías Vol. XXVIII No. 8, pp. 25-31 (2005)

17.Pearl, J.: Bayesian networks, causal inference and knowledge discovery. UCLA Cognitive Systems Laboratory, Technical Report (2001)

18.Puga, J. L., García, J. G., De la Fuente Sánchez, L., De la Fuente Solana, E. I.: Bayesian nets as modelling in psychology. Anales de Psicología/Annals of Psychology, 23(2), 307-316 (2007)

19.Santesmases, M.: Marketing, conceptos y estrategias. 4a . ed., Pirámide. Madrid, España (1999)

20.Spirtes, P., Glymour, C., Scheines, R., Kauffman, S., Aimale, V., Wimberly, F.: Constructing Bayesian network models of gene expression networks from microarray data (2000) 